\title{
Urinary bladder diverticulum as an unusual content of the inguinal canal
}

\author{
F. Burdan 1, 2, E. Starosławska1 , A. Mocarska1 , M. Cendrowska-Pinkosz², M. Caban¹, \\ I. Żelazowska-Cieślińska1, T. Kubiatowski ${ }^{1}$ \\ ${ }^{1}$ St. John's Cancer Centre, Lublin, Poland \\ ${ }^{2}$ Department of Anatomy, Medical University of Lublin, Poland
}

[Received 29 May 2014; Accepted 9 June 2014]

\begin{abstract}
The inguinal urinary bladder hernia is a rare pathology observed mostly in males. A new case of asymptomatic reducible acquired inguinal hernia was revealed in a 54-year-old male during computed tomography (CT) undertaken for oncological follow-up. The right nephrectomy was previously performed due to clear cell carcinoma. The hernia was not visible on the CT 6 months before and on ultrasound performed after voiding. Slight herniation with a wide invagination of transversalis fascia but with empty bladder was seen on CT 4 months after the initial detection of hernia. (Folia Morphol 2014; 73, 4: 500-503)
\end{abstract}

Key words: inguinal bladder hernia, abdominal hernia, inguinal canal, urinary bladder, scrotal cystocoele

\section{INTRODUCTION}

The inguinal canal is an oblique passage that connects the abdominal cavity with the scrotum or internal aspect of labia majora. It is located superior to the medial half of the inguinal ligament, which forms its inferior wall. The aponeurosis of the internal oblique and transverse abdominal muscles is located above, while the aponeurosis of external oblique muscle and transversalis fascia form the anterior and posterior walls, respectively. The proximal opening, known as a deep inguinal ring, is located laterally to the inferior epigastric artery and is the beginning of invagination in the transversalis fascia pushed down in a late stage of the prenatal period by the descending testis. The fascia forms the internal spermatic fascia that covers the wall of the canal. The distal opening of the canal, known as superficial inguinal ring, is surrounded mostly by fibres of the external oblique aponeurosis that form its medial and lateral crus. The superolateral margin is formed by horizontally located fibres (intercrural fibres) which belong to superficial layer of the deep investing fascia overlaying the external oblique muscle and its aponeurosis. Finally, the inferomedial margin is formed by the inguinal falx. The deep inguinal ring is posteriorly covered by the parietal layer of peritoneum, which on the inner surface of anterior abdominal wall forms the lateral inguinal fossa, while superficial inguinal ring is located more medially at the level of the medial umbilical fossa. Between them there is the lateral umbilical fold (elevation of peritoneum at the level of the inferior epigastric vessels), whereas medial margin of the medial inguinal fossa is formed by the medial umbilical folds (elevation of peritoneum at the level of the medial umbilical ligament). Supravesical fossa, located between medial and median umbilical folds, in contrast to above presented ones, is an unlikely site for hernia because its anterior wall is formed mostly by the rectus abdominal muscle $[5,7]$.

In males, the inguinal canal starts to develop by $28^{\text {th }}$ gestational week when a testis reaches the level of the deep inguinal ring. After 3 days, due to its continuous descent, the organ pushes down the anterior

Address for correspondence: Prof. F. Burdan, MD, PhD, ERT, Human Anatomy Department, Medical University of Lublin, ul. Jaczewskiego 4 , 20-059 Lublin, Poland; Radiology Department, St. John's Cancer Centre, ul. Jaczewskiego 7, 20-090 Lublin, Poland, e-mail: fb3@wp.pl 
abdominal wall and exits abdominal cavity, leaving the inguinal canal. In physiological condition the canal contains ductus deferens and its vessels, testicular artery and lymphatic vessels, pampiniform venous plexus, cremaster muscle and its vessels, genital branch of genitofemoral nerve and testicular autonomic plexus. It also contains peritoneal process (processus vaginalis) whose stalk degenerates and separates the proper peritoneal cavity and tunica vaginalis that surround the testis at the end of the pregnancy. In females, the main content of the canal is the round ligament of the uterus and concomitant vessels and nerves. In pathological conditions, especially in early postnatal period, secondary to continuous passage through the processus vaginalis the testis or less commonly, the ovary could be visible. In adults, as a consequence of the increase of intra-abdominal pressure also viscera, especially small intestinal loops and greater omentum, may exit the abdominal cavity [5].

In the current paper a rare case of the urinary bladder inguinal herniation is presented.

\section{CASE DESCRIPTION}

A 54-year-old male underwent the second regular follow-up computed tomography (CT) of chest, abdomen and pelvis. His right kidney was surgically removed 1 year before due to renal clear cell carcinoma (Fuhrman 1, $\mathrm{pT}_{2 \mathrm{~A}} \mathrm{~N}_{0} \mathrm{M}_{1}$ ). No radiotherapy was performed. However, due to skeletal and lung metastases, the patient was treated with pazopanib which is a potent and selective multi-targeted receptor tyrosine kinase inhibitor. The medical history was otherwise unremarkable, without any pathological urogenital symptoms or voiding complications present at the time of investigation.

The morphology of all the internal organs and skeleton was similar to the previous CT that was undertaken 6 months previously. However, a $2.5 \mathrm{~cm}$ long diverticulum of the urinary bladder penetrating to the right inguinal canal at the level of the medial inguinal fossa was found (Figs. 1, 2). Similar but much smaller structure was visible on the left side of the organ as well. Both processes were the only abnormalities of the bladder. The pathology was not present in previous $\mathrm{CT}$ and ultrasound examination performed after voiding. However, slight herniation with a wide invagination of transversalis fascia but with empty bladder was seen on CT 4 months later.

The abnormality was classified as the reducible, acquired direct inguinal hernia of the urinary bladder.

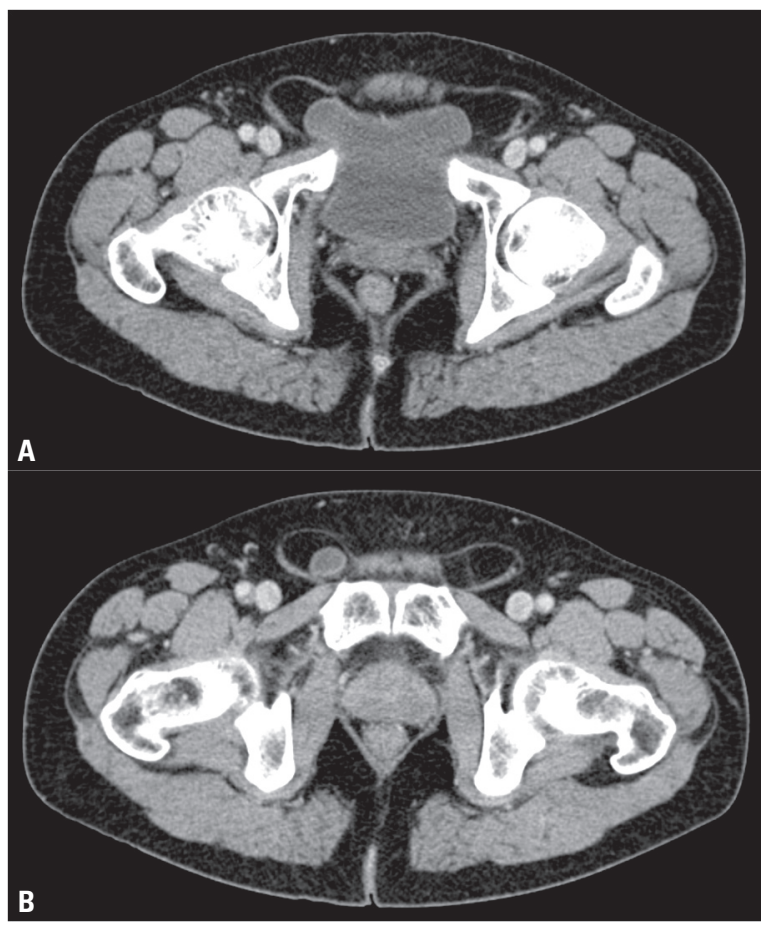

Figure 1. Axial scans of computed tomography with a well-formed right direct inguinal hernia of the urinary bladder over $(\mathbf{A})$ and below (B) the medial inguinal fossa. Elongation of the left side of the anterior wall of the organ is also visible (B).

No form of treatment for hernia was administered or recommended.

\section{DISCUSSION}

The inguinal bladder hernia was described for the first time by Levine in 1951 as a scrotal cystocoele [4]. It is a rare pathological condition with high predominance in males, observed in $1-4 \%$ of all hernias in that location [11]. However, much higher incidence $(10 \%)$ was revealed in men aged over 50 [3].

Since small hernias - like the one in the reported case - are asymptomatic, most of them are incidental findings reported in various radiological examinations taken for unrelated medical reason. Even symptomatic ones are rarely diagnosed preoperatively $(<7 \%)[3,8]$. Patients with a massive bladder penetration may presents intermittent swelling in the groin and scrotum or labia majora, as well as various lower urinary symptoms like pollakiuria, hematuria, nocturia and renal failure that requires nephrostomy to reduce the urinary tract decompression [12]. The last complication is probably secondary to the ureter obstruction caused by abnormal position of the bladder. Usually patients have to compress external 

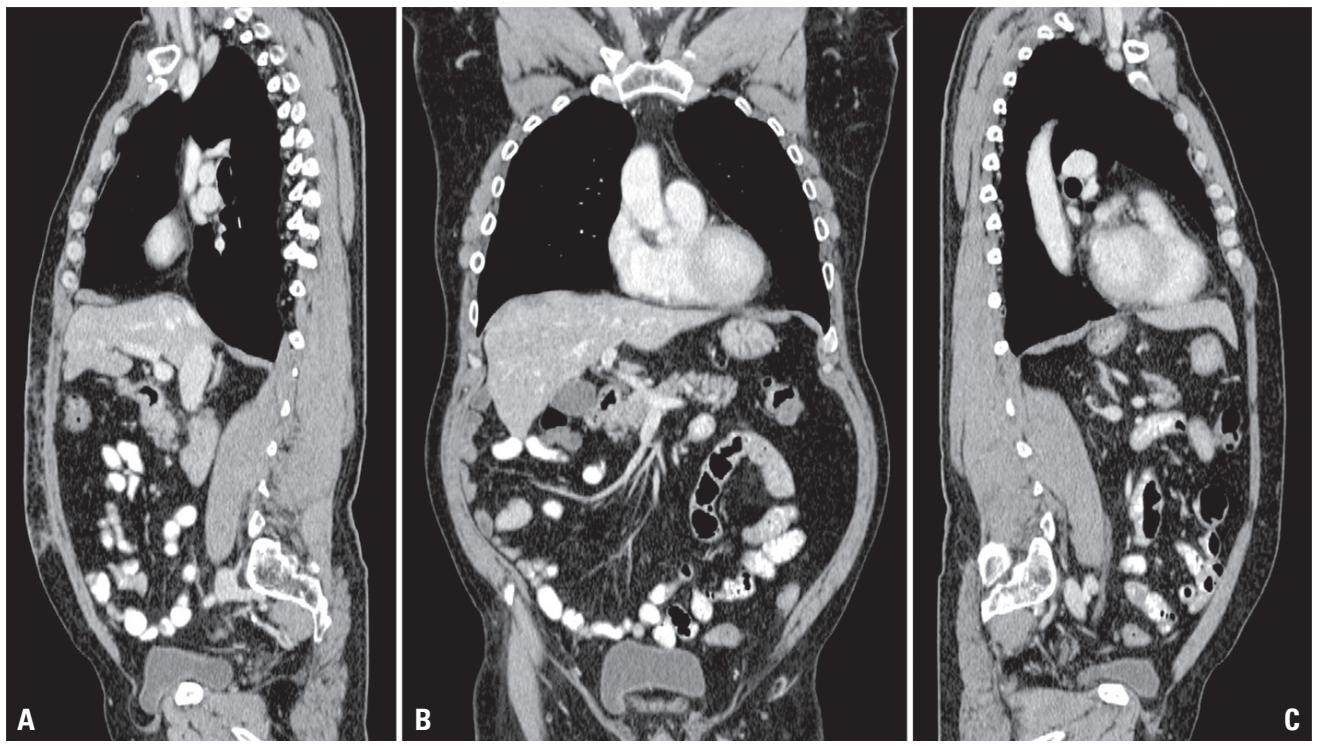

Figure 2. Sagittal (A, C) and coronal (B) scans of computed tomography with a well-formed direct inguinal hernia of the urinary bladder on the right side $(\mathbf{A}, \mathbf{B})$ and slight elongation on the left side $(\mathbf{C})$.

genital organs during voiding but this process may be also complicated by micturition - at the beginning urination is spontaneous but the second step involves a massage. In most severe cases, patients usually related all symptoms to concomitant prostate enlargement and/or cystitis [6].

The main risk factors for the bladder inguinal hernia are obesity, increased abdominal pressure, chronic urinary obstruction, decreased bladder tone, previous herniorrhaphy, as well as weakness of abdominal and pelvic muscles $[1,3,8]$. In case of incisional bladder hernia, a previous therapy with steroids, diabetes mellitus, chronic obstructive pulmonary disease, inappropriate technical procedure used for wound closure during previous operations and postoperative complications like wound infections and ileus have been postulated [10]. Irrespective of the contents of the inguinal canal, the treatment is identical and usually involves a surgical mesh placement. However, in severe herniation the nephrostomy may be required. Bladder resection is performed only in case of severe complications like necrosis or a true herniated bladder diverticulum, a tight hernia neck or tumour in the herniated bladder [6].

For a long time the gold standard in inguinal bladder hernia diagnosis was a cystography but currently a multidetector CT seems to be more appropriate, since it gives better information on surrounding structures and morphology of anterior abdominal wall. As it was done in our case, the use of multiplanar reconstruction during postprocessing gives possibility to present a direct connection of pathological structure with the bladder. Moreover, such techniques may also be helpful in evaluating other parts of the urinary tract. As the presented case, it is especially important since association of the hernia with bladder or other urological malignancies has been described $[2,8,9]$. However, the pathology could be also diagnosed during the ultrasound examination using sector or linear probe [6].

It is important to state that complications of inguinal bladder hernia include vesico-ureteric reflux, hydronephrosis, bladder rupture and infarction, as well as strangulation [6].

\section{CONCLUSIONS}

The reported reducible acquired direct inguinal hernia of the urinary bladder diverticulum is a rare pathology observed mostly in males. Its presence requires detailed urinary tract examination due to high coexistence with various malignancies.

\section{REFERENCES}

1. Bisharat M, O'Donnell ME, Thompson T, MacKenzie N, Kirkpatrick D, Spence RA, Lee J (2009) Complications of inguinoscrotal bladder hernias: a case series. Hernia, 13: 81-84.

2. Darío Casas J, Mariscal A, Gallart A (1997) Bladder cancer within an inguinoscrotal bladder hernia. Am J Roentgenol, 169: 1194-1195.

3. Gomella LG, Spires SM, Burton JM, Ram MD, Flanigan RC (1985) The surgical implications of herniation of the urinary bladder. Arch Surg, 120: 964-967. 
4. Levine B (1951) Scrotal cystocele. J Am Med Assoc, 147: 1439-1441.

5. Moore KL, Dalley AF ed. (2006) Clinically oriented anatomy. $5^{\text {th }}$ Ed. Lippincott Williams \& Wilkins, Philadelphia.

6. Moufid K, Touiti D, Mohamed L (2013) Inguinal bladder hernia: four case analyses. Rev Urol, 15: 32-36.

7. Narkiewicz O, Moryś J ed. (2010) Anatomia człowieka. Wydawnictwo Lekarskie PZWL, Warsawa.

8. Oruç MT, Akbulut Z, Ozozan O, Coşkun F (2004) Urological findings in inguinal hernias: a case report and review of the literature. Hernia, 8: 76-79.

9. Pastor Navarro H, Martínez Ruiz J, Carrión Lopez P, Martínez Sanchíz C, Lorenzo Romero JG, Cañamares
Pabolaza L, Polo Ruiz L, Pastor Guzman JM, García Blazquez E, Virseda Rodríguez JA (2010) Tumor inside an inguinoscrotal bladder hernia. Arch Esp Urol, 63: 471-476.

10. Sasaki S, Miura E, Nakayama H, Watanabe T (2014) An incisional bladder hernia following appendectomy: report of a case. Surg Today, 44: 1953-1956.

11. Thompson JE Jr, Taylor JB, Nazarian N, Bennion RS (1986) Massive inguinal scrotal bladder hernias: a review of the literature with 2 new cases. J Urol, 136: 1299-1301.

12. Wagner AA, Arcand P, Bamberger MH (2004) Acute renal failure resulting from huge inguinal bladder hernia. Urology, 64: 156-157. 